\title{
Correction to: A new three-level fault tolerance arithmetic and logic unit based on quantum dot cellular automata
}

\author{
Mahya Rahimpour Gadim ${ }^{1}$ - Nima Jafari Navimipour ${ }^{1}$ (B)
}

Published online: 18 January 2018

(C) Springer-Verlag GmbH Germany, part of Springer Nature 2018

\section{Correction to: Microsyst Technol \\ https://doi.org/10.1007/ \\ s00542-017-3502-x}

Unfortunately, the Fig. 12 was incorrectly published in the original article. Hereby, the correct figure is given below:

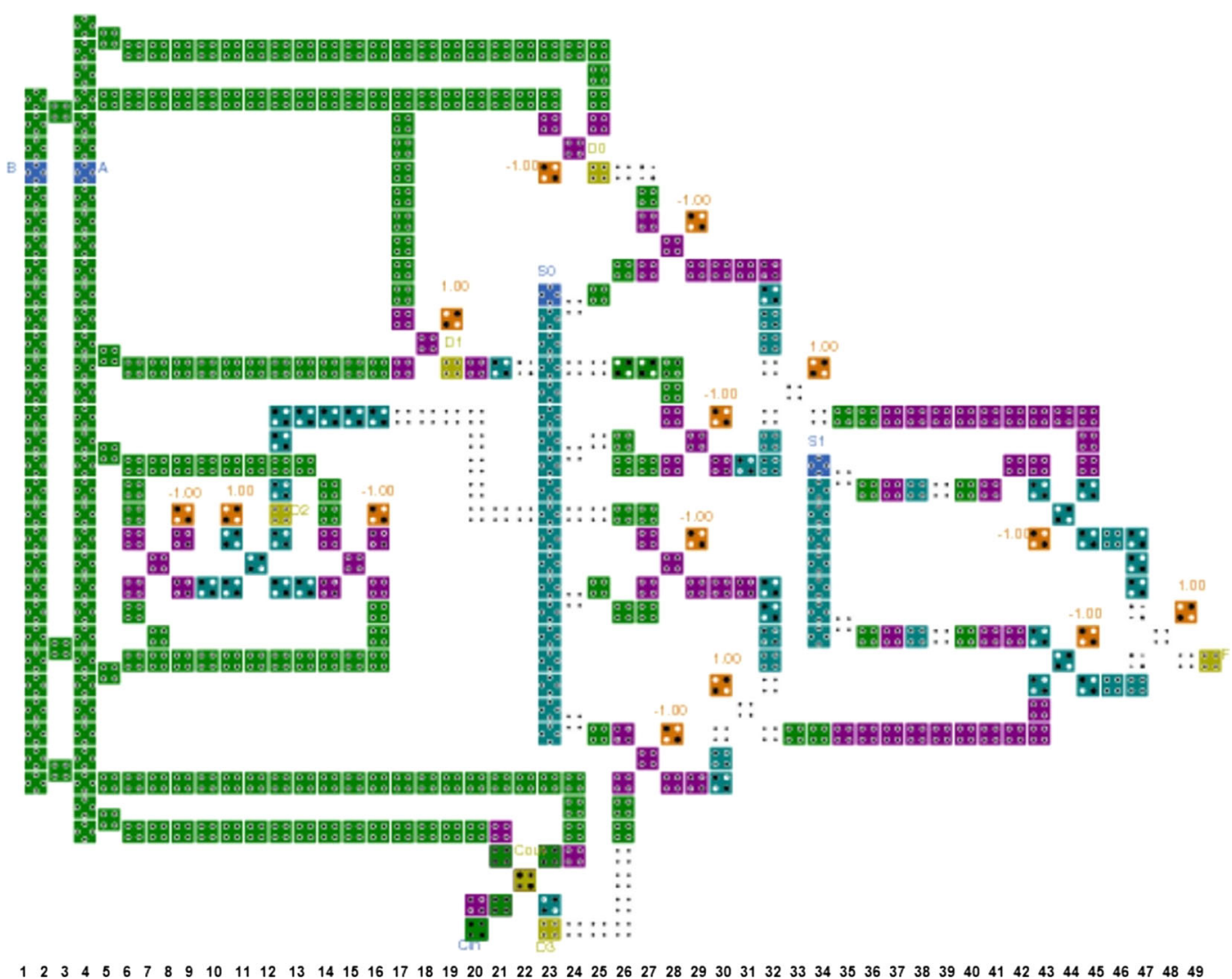

The original article can be found online at https:// doi.org/10.1007/s00542-017-3502-x.

Nima Jafari Navimipour

jafari@iaut.ac.ir; n.jafari.n@gmail.com

1 Department of Computer Engineering, Tabriz Branch,

Islamic Azad University, Tabriz, Iran 